\title{
Alternative diagnoses and demographics associated with a raised quantitative faecal immunochemical test in symptomatic patients
}

\author{
Mark S Johnstone' ${ }^{(0,}$, Gillian Miller', Grace Pang', Paul Burton², Georgios Kourounis', \\ Jack Winter ${ }^{3}$, Emilia Crighton ${ }^{4}$, David Mansouri ${ }^{5}$, Paul Witherspoon ${ }^{6}$, Karen Smith ${ }^{7}$ and \\ Stephen T McSorley'
}

\begin{abstract}
Background: The faecal immunochemical test (FIT) has proven utility for colorectal cancer detection in symptomatic patients. However, most patients with a raised faecal haemoglobin $(\mathrm{f}-\mathrm{Hb})$ do not have colorectal cancer. We investigated alternative diagnoses and demographics associated with a raised $\mathrm{f}-\mathrm{Hb}$ in symptomatic patients.

Methods: A retrospective, observational study was performed of patients with FIT submitted between August 2018 to January 2019 in NHS Greater Glasgow and Clyde followed by colonoscopy. Colonoscopy/pathology reports were searched for alternative diagnoses. Covariables were compared using the $\chi^{2}$ test. Multivariate binary logistic regression identified independent predictors of a raised $\mathrm{f}-\mathrm{Hb}$.

Results: I 272 patients were included. In addition to colorectal cancer (odds ratio (OR), 9.27 (95\% confidence interval (Cl): 3.6I-23.83; $p<0.00 \mathrm{I})$ ), older age (OR, I.52 (95\% Cl: I.00-2.32; $p=0.05)$ ), deprivation (OR, I.54 (95\% Cl: I.2I-I.94; $p<$ $0.00 \mathrm{I})$ ), oral anticoagulants (OR, I.78 (95\% Cl: I.0I-3.I5; $p=0.046)$ ), rectal bleeding (OR, I.47 (95\% Cl: I.I5-I.88; $p=$ $0.002)$ ), advanced adenoma (OR, 7.52 (95\% Cl: 3.90-14.49; $p<0.00 \mathrm{I})$ ), non-advanced polyps (OR, I.78 (95\% Cl: I.332.38; $p<0.00 \mathrm{I})$ ) and inflammatory bowel disease (IBD) (OR, 4.19 (95\% Cl: 2. I7-8.07; $p<0.00 \mathrm{I})$ ) independently predicted raised f-Hb. Deprivation (Scottish Index of Multiple Deprivation (SIMD) I-2: OR, 2.13 (95\% Cl: I.38-3.29; $p=0.00 \mathrm{I}$ )) independently predicted a raised $\mathrm{f}-\mathrm{Hb}$ in patients with no pathology found at colonoscopy.

Conclusions: An elevated $\mathrm{f}-\mathrm{Hb}$ is independently associated with older age, deprivation, anticoagulants, rectal bleeding, advanced adenoma, non-advanced polyps and IBD in symptomatic patients. Deprivation is associated with a raised $\mathrm{f}-\mathrm{Hb}$ in the absence of pathology. This must be considered when utilising FIT in symptomatic patients.
\end{abstract}

Keywords

Faecal, immunochemical, test, haemoglobin, colorectal, cancer, symptomatic

Accepted: I0th January 2022

\footnotetext{
'Academic Unit of Surgery, School of Medicine, University of Glasgow, Glasgow, UK

${ }^{2}$ eHealth, Corporate Services, Business Intelligence, NHS Greater Glasgow and Clyde, Glasgow, UK

${ }^{3}$ Department of Gastroenterology, Glasgow Royal Infirmary, NHS Greater Glasgow and Clyde, Glasgow, UK

${ }^{4}$ Public Health, Health Service, Public Health Screening, NHS Greater Glasgow and Clyde, Glasgow, UK

${ }^{5}$ Department of Coloproctology, Glasgow Royal Infirmary, NHS Greater Glasgow and Clyde, Glasgow, UK

${ }^{6}$ Department of Colorectal Surgery, Queen Elizabeth University Hospital, NHS Greater Glasgow and Clyde, Glasgow, UK

${ }^{7}$ Department of Clinical Biochemistry, Glasgow Royal Infirmary, NHS Greater Glasgow and Clyde, Glasgow, UK
}

Corresponding author:

Mark S Johnstone, Academic Unit of Surgery, School of Medicine, University of Glasgow, New Lister Building, Glasgow Royal Infirmary, 8-16 Alexandra Parade, Glasgow G3I 2ER, UK.

Email: mark.johnstone.2@glasgow.ac.uk. 


\section{Introduction}

The faecal immunochemical test (FIT) has proven utility for colorectal cancer detection in symptomatic patients, sensitivity and specificity reportedly ranging from $85 \%$ to $100 \%$ and $56 \%-91 \%$, respectively, at a threshold of $\geq 10 \mu \mathrm{g}$ $\mathrm{Hb} / \mathrm{g}$ faeces. ${ }^{1-8}$ The National Institute for Health and Care Excellence (NICE) now recommends FIT be used in patients with high-risk symptoms that may trigger an urgent suspected cancer referral $(\mathrm{NG} 12)^{9}$ and in those with lower risk symptoms (DG30). ${ }^{10}$ In response, a number of UK health boards/trusts have introduced universal FIT submission as part of symptomatic lower GI referral pathways. ${ }^{11}$ However, most patients with a raised $\mathrm{f}-\mathrm{Hb}$ will not have a colorectal cancer. A raised $\mathrm{f}-\mathrm{Hb}$ in symptomatic patients has been correlated with advanced adenomas and inflammatory bowel disease. ${ }^{12-15}$ Indeed, there is evidence that FIT can be used as a marker of disease activity in ulcerative colitis ${ }^{16-20}$ and colonic Crohn's ${ }^{21}$ as an adjunct to faecal calprotectin. Additionally, higher FIT positivity in the context of bowel cancer screening has been independently associated with older age, male sex, deprivation, aspirin, non-steroidal anti-inflammatory drugs (NSAIDs), oral anticoagulants, proton pump inhibitors (PPIs), antibiotics and smoking, ${ }^{22-25}$ and false positivity has been related to younger age, female sex, smoking, high BMI, successive screening, aspirin, NSAIDs, PPIs, antibiotics, laxatives, non-advanced adenomas, diverticular disease, haemorrhoids, anal fissures and peptic ulceration. ${ }^{25-32}$

To date, no studies have examined demographics which independently predict a raised $\mathrm{f}-\mathrm{Hb}$ in symptomatic patients and very few have explored non-cancer diagnoses which correlate with $\mathrm{f}-\mathrm{Hb}$. We aimed to establish demographics and alternative pathologies associated with a raised $\mathrm{f}-\mathrm{Hb}$ in a cohort of symptomatic patients.

\section{Methods}

A retrospective, observational study was conducted to include all adult ( $\geq 16$ years) patients with an FIT submitted from primary care between August 2018 and January 2019 in NHS Greater Glasgow and Clyde (period in which FIT was introduced to local referral pathways).

\section{Faecal immunochemical test specimen collection and handling}

FIT collection kits containing a single FIT collection device (EXTEL HEMO AUTO MC Collection Picker, Minaris Medical Co, Ltd, Tokyo, Japan, supplied by Alpha Labs Ltd, Eastleigh, Hants, UK) with accompanying pictorial instructions, and return envelopes were supplied to general practitioners (GPs) as an adjunct to guide symptomatic lower gastrointestinal (GI) referrals. The collection device is in the form of a picker with an internal septum which removes excess faeces and provides a consistent 2-mg sample, which is inserted into a vial containing $2 \mathrm{~mL}$ of buffer following collection. Patients were asked to collect a single faecal sample and return to their GP practice as soon as possible. The kits were transported at ambient temperature via routine specimen collection services and stored at $4^{\circ} \mathrm{C}$ prior to analysis in a single centralised laboratory (Stobhill Hospital, Glasgow).

\section{Faecal immunochemical test analysis}

Analysis was carried out on a HM-KACKarc system (Minaris Medical Co, Ltd), once for each sample, Monday to Friday so most samples were analysed on day of receipt. The manufacturers quote a limit of detection of $2 \mu \mathrm{g} / \mathrm{g}$, a limit of quantification of $7 \mu \mathrm{g} / \mathrm{g}$ and an upper measurement limit of $400 \mu \mathrm{g} / \mathrm{g}$. Specimens with $\mathrm{f}-\mathrm{Hb}$ concentrations above this limit were not diluted and re-analysed.

\section{Faecal immunochemical test result quality management}

All biomedical science staff are Health Care and Professionals Council (HCPC) registered and undergo training and local competency assessment prior to operating the HM-KACKarc analyser. The analyser is calibrated daily. There are two internal quality controls (IQCs): EXTEL HEMO AUTO HS Low IQC and EXTEL HEMO AUTO HS High IQC. West guard rule criteria are used for acceptance or rejection of analytical runs. IQC performance is reviewed monthly and manufacturers' targets are refined when appropriate. Current performance: low QC mean = $23.2 \mu \mathrm{g} / \mathrm{g}, \mathrm{CV}=8.3 \%$, high QC mean $=90.7 \mu \mathrm{g} / \mathrm{g}$ and $\mathrm{CV}$ $6.6 \%$. The laboratory participates in external quality assessment via the United Kingdom National External Quality Assessment Service (UK NEQAS) on a monthly basis, with good recent performance scores.

\section{Faecal immunochemical test result handling}

Faecal immunochemical test results are electronically transferred from the analyser into the Laboratory Information Management System (LIMS) and patient record. Any result with an error code is investigated and the appropriate result entered manually. Finally, results are electronically reported to the requesting GP with a range of $\leq 9 \mu \mathrm{g} / \mathrm{g}$ to $\geq 400 \mu \mathrm{g} / \mathrm{g}$. Samples $\geq 10 \mu \mathrm{g} / \mathrm{g}$ were defined as raised as per the NICE DG30 guidance. ${ }^{10}$

\section{Patient identification and data collection}

To identify study participants, a search of the clinical biochemistry repository was conducted. To obtain patient demographics and outcomes, cross-referencing of the SCI 
Store, SCI Gateway, Unisoft, Clinical Portal and Managed Clinical Networks (MCN) Cancer Registry was performed with the community health index (CHI) number used as the linkage variable. Caldicott guardian approval was given by NHS GG\&C to safeguard the record linkage with ethical approval waived for the purposes of service development. A search of SCI store (Scottish Care Information Store Version 8.5) allowed identification of patient demographics and blood results. SCI Gateway (Scottish Care Information Gateway R 20.0) was searched to identify referral letters from primary care to general surgery or gastroenterology within 6 weeks of FIT collection. These letters were manually screened to identify lower GI symptoms and coded as rectal bleeding, persistent diarrhoea, other change in bowel habit, weight loss, abdominal pain, anal pain, faecal soiling, rectal mass and abdominal mass. Referral letters also identified patient co-morbidity. Unisoft (Unisoft Medical Systems GI Reporting Tool) identified all patients who underwent a colonoscopy following FIT collection. Each colonoscopy record and any accompanying pathology records were screened manually to identify lower GI diagnoses and coded as cancer, advanced adenoma(s), any advanced polyp(s), non-advanced polyp(s), inflammatory bowel disease, other inflammation (infective colitis, collagenous colitis, lymphocytic colitis and inflammatory polyps), diverticulosis, haemorrhoids, angiodysplasia/ telangiectasia, radiation proctitis, other malignancy (anal squamous cell carcinoma and rectal lymphoma), melanosis coli, anal fissure or fistula, rectal prolapse, fibroepithelial anal polyp and lipoma. Advanced adenomas were defined as those $\geq 10 \mathrm{~mm}$ or with the presence of high-grade dysplasia. Advanced polyps were defined as advanced adenomas or advanced serrated polyps $\geq 10 \mathrm{~mm}$ or with the presence of any grade of dysplasia as per The British Society of Gastroenterology/Association of Coloproctology of Great Britain and Ireland surveillance guidelines. ${ }^{33}$ The $\mathrm{MCN}$ cancer registry was searched to identify all new diagnoses of colorectal cancer up to November 2020. For the purposes of analysis, patients were divided into those with significant bowel disease (cancer, advanced adenoma, advanced polyp, $\geq 5$ non-advanced polyps or inflammatory bowel disease), other bowel disease (any other positive finding at colonoscopy) and no pathology (entirely normal colonoscopy).

\section{Statistical analysis}

FIT results were grouped by $\mathrm{f}-\mathrm{Hb}$ concentrations of $<10 \mu \mathrm{g} / \mathrm{g}$, $10-149 \mu \mathrm{g} / \mathrm{g}, 150-399 \mu \mathrm{g} / \mathrm{g}$ and $>399 \mu \mathrm{g} / \mathrm{g} .{ }^{34}$ Patients were defined as anaemic (male haemoglobin $(\mathrm{Hb})<130 \mathrm{mg} / \mathrm{L}$ and female $\mathrm{Hb}<120 \mathrm{mg} / \mathrm{L}$ ) and iron deficient (ferritin $<15 \mu \mathrm{g} / \mathrm{L}$ ) based on World Health Organisation (WHO) guidelines. ${ }^{35}$ Covariables were compared using crosstabulation and the $\chi^{2}$ test or Fisher's exact test. A value of $p<0.05$ was considered statistically significant. To identify covariables which independently predicted a raised $\mathrm{f}-\mathrm{Hb}$, univariate followed by multivariate binary logistic regression was performed. Covariables of interest from the $\chi^{2}$ analysis were carried into the regression analysis. Variables found to be significant on $\chi^{2}$ analysis but where there were insufficient numbers for regression analysis were excluded. For the purposes of regression analysis, FIT was converted to a binary variable: normal (f- $\mathrm{Hb}<10 \mu \mathrm{g} / \mathrm{g}$ ) vs. raised (f- $\mathrm{Hb} \geq 10 \mu \mathrm{g} / \mathrm{g}$ ). This allowed calculation of odds ratios (ORs) and 95\% confidence intervals $(95 \% \mathrm{CI})$. Covariables significant on univariate analysis $(p<0.05)$ were entered into a multivariate model using the backwards conditional method in which variables with a significance of $p<0.1$ were removed in a stepwise fashion. The same process was then performed in turn only for those patients with significant bowel disease, other bowel disease and no pathology. Statistical analysis was performed using SPSS software (SPSS Inc., Chicago, Illinois, USA).

\section{Results}

4968 patients had a FIT sample submitted from primary care between August 2018 and January 2019 in NHS GG\&C. Of these, 2434 patients were subsequently referred to general surgery or gastroenterology and 1327 of those underwent colonoscopy. Of those who underwent colonoscopy, 572 (43.1\%) had f-Hb $<10 \mu \mathrm{g} / \mathrm{g}$ and $700(52.8 \%) \mathrm{f}-\mathrm{Hb} \geq 10 \mu \mathrm{g} /$ $\mathrm{g}$, with $430(32.4 \%)$ between 10 and $149 \mu \mathrm{g} / \mathrm{g}, 89(6.7 \%)$ between 150 and $399 \mu \mathrm{g} / \mathrm{g}$ and $181(13.6 \%) \geq 400 \mu \mathrm{g} / \mathrm{g}$. 55 (4.1\%) samples could not be processed by the laboratory due to faecal contamination, expired collection device or insufficient patient identification, and were not repeated. These patients were excluded from the final analysis, leaving a total of 1272 patients who underwent colonoscopy and had a valid FIT.

Median age of these 1272 patients was 60 years (range, 17-94), with 558 (43.9\%) male and 714 (56.1\%) females. $561(44.1 \%)$ patients reported rectal bleeding; $348(27.4 \%)$, persistent diarrhoea; $602(47.3 \%)$, other change in bowel habit; 214 (16.8\%), weight loss; 383 (30.1\%) abdominal pain; $33(2.6 \%)$, anal pain; $77(6.1 \%)$, faecal soiling; 25 $(2.0 \%)$, rectal mass; and 31 (2.4\%), abdominal mass.

\section{Comparison of demographics by $\mathrm{f}-\mathrm{Hb}$ concentration}

Table 1 shows a comparison of demographics by $\mathrm{f}-\mathrm{Hb}$ concentration. Having a raised $\mathrm{f}-\mathrm{Hb}$ was associated with either being below ( $<50$ years) or above $(\geq 75$ years) the Scottish Bowel Screening Programme age (50-74 years) $(p<0.001)$. There was no association between $\mathrm{f}-\mathrm{Hb}$ and sex $(p=0.08)$. Deprivation was associated with a higher $\mathrm{f}-\mathrm{Hb}$ $(p=0.004)$. No individual co-morbidity was associated with a raised $\mathrm{f}-\mathrm{Hb}$; however, the presence of diabetes $(p=0.015)$ or hypertension $(p=0.004)$ seemed to be mildly protective. Patients on oral anticoagulants or PPIs were significantly 
Table I. Comparison of demographics by $\mathrm{f}-\mathrm{Hb}$ concentration.

\begin{tabular}{|c|c|c|c|c|c|c|c|}
\hline \multirow[b]{3}{*}{$N$} & & \multirow[b]{2}{*}{ All } & \multicolumn{4}{|c|}{$\mathrm{f}-\mathrm{Hb}$ ( $\mu \mathrm{g} \mathrm{Hb/g}$ faeces) } & \multirow{3}{*}{$p$} \\
\hline & & & \multirow{2}{*}{$\frac{<10}{572}$} & \multirow{2}{*}{$\frac{10-149}{430}$} & \multirow{2}{*}{$\frac{150-399}{89}$} & \multirow{2}{*}{$\frac{\geq 400}{181}$} & \\
\hline & & 1272 & & & & & \\
\hline \multirow[t]{4}{*}{ Age (years) } & Median (range) & $60(17-94)$ & $58(17-88)$ & $63(19-92)$ & 56 (19-94) & $56(17-90)$ & $<0.001$ \\
\hline & $<50$ & $318(25.0 \%)$ & 142 (24.8\%) & $82(19.1 \%)$ & $32(36.0 \%)$ & $62(34.3 \%)$ & \\
\hline & $50-74$ & 759 (59.7\%) & $370(64.7 \%)$ & $259(60.2 \%)$ & 44 (49.4\%) & $86(47.5 \%)$ & \\
\hline & $\geq 75$ & 195 (I5.3\%) & 60 (10.5\%) & 89 (20.7\%) & $13(14.6 \%)$ & $33(18.2 \%)$ & \\
\hline \multirow[t]{2}{*}{ Sex } & Male & $558(43.9 \%)$ & $235(41.1 \%)$ & 188 (43.7\%) & 48 (53.9\%) & $87(48.1 \%)$ & 0.080 \\
\hline & Female & $714(56.1 \%)$ & 337 (58.9\%) & $242(56.3 \%)$ & 41 (46.1\%) & $94(51.9 \%)$ & \\
\hline \multirow[t]{2}{*}{$\begin{array}{l}\text { Scottish Index of Multiple } \\
\text { Deprivation }\end{array}$} & $\begin{array}{l}\text { Non-deprived (SIMD } \\
3-5)\end{array}$ & $627(49.3 \%)$ & $314(54.9 \%)$ & 193 (44.9\%) & 37 (4I.6\%) & $83(45.9 \%)$ & 0.004 \\
\hline & Deprived (SIMD I-2) & 645 (50.7\%) & 258 (45.1\%) & 237 (55.1\%) & $52(58.4 \%)$ & $98(54.1 \%)$ & \\
\hline \multirow[t]{9}{*}{ Co-morbidity } & Asthma & $163(12.8 \%)$ & 71 (12.4\%) & 59 (I3.7\%) & 14 (I5.7\%) & $19(10.5 \%)$ & 0.584 \\
\hline & COPD & $76(6.0 \%)$ & $31(5.4 \%)$ & $38(8.8 \%)$ & $5(5.6 \%)$ & 2 (I.I\%) & 0.003 \\
\hline & Diabetes & 157 (12.3\%) & $82(14.3 \%)$ & $57(13.3 \%)$ & $6(6.7 \%)$ & $12(6.6 \%)$ & 0.015 \\
\hline & Hypertension & $109(8.6 \%)$ & 65 (II.4\%) & $33(7.7 \%)$ & $4(4.5 \%)$ & $7(3.9 \%)$ & 0.004 \\
\hline & IHD & 150 (II.8\%) & $60(10.5 \%)$ & $61(14.2 \%)$ & $9(10.1 \%)$ & $20(11.0 \%)$ & 0.303 \\
\hline & Cerebrovascular disease & $42(3.3 \%)$ & $27(4.7 \%)$ & $9(2.1 \%)$ & $2(2.2 \%)$ & $4(2.2 \%)$ & 0.087 \\
\hline & PVD & $9(0.7 \%)$ & $4(0.7 \%)$ & $2(0.5 \%)$ & $2(2.2 \%)$ & I (0.6\%) & 0.331 \\
\hline & IBD (prior diagnosis) & $6(0.5 \%)$ & $3(0.5 \%)$ & $2(0.5 \%)$ & $0(0.0 \%)$ & I (0.6\%) & 0.923 \\
\hline & CRC (prior diagnosis) & $8(0.6 \%)$ & $3(0.5 \%)$ & $5(1.2 \%)$ & $0(0.0 \%)$ & $0(0.0 \%)$ & 0.287 \\
\hline \multirow[t]{10}{*}{ Medication } & Aspirin & $268(21.1 \%)$ & II 6 (20.3\%) & $100(23.3 \%)$ & $18(20.2 \%)$ & $34(18.8 \%)$ & 0.561 \\
\hline & NSAIDs & 166 (13.1\%) & 77 (13.5\%) & 49 (II.4\%) & 14 (I5.7\%) & $26(14.4 \%)$ & 0.580 \\
\hline & Clopidogrel/Ticagrelor & $58(4.6 \%)$ & $26(4.5 \%)$ & $18(4.2 \%)$ & $5(5.6 \%)$ & $9(5.0 \%)$ & 0.932 \\
\hline & Oral anticoagulants & $69(5.4 \%)$ & $20(3.5 \%)$ & $26(6.0 \%)$ & $9(10.1 \%)$ & $14(7.7 \%)$ & 0.017 \\
\hline & ACE inhibitors & $259(20.4 \%)$ & 124 (21.7\%) & $86(20.0 \%)$ & 14 (I5.7\%) & $35(19.3 \%)$ & 0.585 \\
\hline & Statins & $364(28.6 \%)$ & 150 (26.2\%) & 134 (31.2\%) & $28(31.5 \%)$ & $52(28.7 \%)$ & 0.345 \\
\hline & PPI & $606(47.6 \%)$ & 257 (44.9\%) & $228(53.0 \%)$ & 48 (53.9\%) & $73(40.3 \%)$ & 0.007 \\
\hline & $\mathrm{H} 2$ antagonists & $39(3.1 \%)$ & $16(2.8 \%)$ & $14(3.3 \%)$ & $5(5.6 \%)$ & $4(2.2 \%)$ & 0.460 \\
\hline & Metformin & $76(6.0 \%)$ & $39(6.8 \%)$ & $26(6.1 \%)$ & $6(6.7 \%)$ & $5(2.8 \%)$ & 0.249 \\
\hline & Anti-spasmodics & $315(24.8 \%)$ & $156(27.3 \%)$ & $98(22.8 \%)$ & $21(23.6 \%)$ & $40(22.1 \%)$ & 0.310 \\
\hline \multirow[t]{9}{*}{ Symptoms } & Rectal bleeding & 561 (44.1\%) & $221(38.6 \%)$ & $160(37.2 \%)$ & 51 (57.3\%) & 129 (7I.3\%) & $<0.001$ \\
\hline & Persistent diarrhoea & 348 (27.4\%) & 195 (34.1\%) & $88(20.5 \%)$ & $21(23.6 \%)$ & $44(24.3 \%)$ & $<0.001$ \\
\hline & $\begin{array}{l}\text { Other change in bowel } \\
\text { habit }\end{array}$ & $602(47.3 \%)$ & $284(49.7 \%)$ & $213(49.5 \%)$ & $42(47.2 \%)$ & $63(34.8 \%)$ & 0.004 \\
\hline & Weight loss & $214(16.8 \%)$ & 108 (18.9\%) & $73(17.0 \%)$ & II (I2.4\%) & $22(12.2 \%)$ & 0.120 \\
\hline & Abdominal pain & 383 (30.1\%) & 189 (33.0\%) & $123(28.6 \%)$ & 27 (30.3\%) & $44(24.3 \%)$ & 0.127 \\
\hline & Anal pain & $33(2.6 \%)$ & $14(2.4 \%)$ & $9(2.1 \%)$ & $2(2.2 \%)$ & $8(4.4 \%)$ & 0.406 \\
\hline & Faecal soiling & 77 (6.1\%) & $5 \mathrm{I}(8.9 \%)$ & 17 (4.0\%) & $6(6.7 \%)$ & $3(1.7 \%)$ & $<0.001$ \\
\hline & Rectal mass & $25(2.0 \%)$ & $17(3.0 \%)$ & $7(1.6 \%)$ & $0(0.0 \%)$ & I (0.6\%) & 0.074 \\
\hline & Abdominal mass & $31(2.4 \%)$ & $12(2.1 \%)$ & $12(2.8 \%)$ & $3(3.4 \%)$ & $4(2.2 \%)$ & 0.833 \\
\hline \multirow[t]{2}{*}{ Anaemia } & Anaemia $^{a}$ & $246(21.8 \%)$ & $106(20.9 \%)$ & 74 (19.6\%) & $22(26.8 \%)$ & $44(26.8 \%)$ & 0.173 \\
\hline & Iron deficiency anaemia ${ }^{\mathrm{b}}$ & 91 (8.1\%) & $46(9.2 \%)$ & $21(5.6 \%)$ & $4(4.9 \%)$ & $20(12.3 \%)$ & 0.032 \\
\hline
\end{tabular}

aMissing data for $14 \mathrm{I}$ (II.1\%) patients.

${ }^{b}$ Data missing for 153 (12.0\%) patients.

more likely to have a raised $\mathrm{f}-\mathrm{Hb}(p=0.017)$ or raised $\mathrm{f}-\mathrm{Hb}$ between 10 and $399 \mu \mathrm{g} / \mathrm{g}(p=0.007)$, respectively. Patients self-reporting rectal bleeding were more likely to have a raised $\mathrm{f}-\mathrm{Hb}(p<0.001)$, while a history of persistent diarrhoea $(p<0.001)$, other change in bowel habit $(p=0.004)$ or faecal soiling $(p<0.001)$ were associated with a lower $\mathrm{f}-\mathrm{Hb}$.

\section{Cancer cases}

With a median 23-month (range, 21-25) follow-up, 54 patients were diagnosed with a colorectal cancer. $5(9.3 \%)$ had a f-Hb $<$ $10 \mu \mathrm{g} / \mathrm{g} ; 9$ (16.7\%), between 10 and $149 \mu \mathrm{g} / \mathrm{g} ; 7$ (13.0\%), between 150 and $399 \mu \mathrm{g} / \mathrm{g}$; and $33(61.1 \%), \geq 400 \mu \mathrm{g} / \mathrm{g}$. 


\section{Alternative colonoscopic diagnoses associated with a raised $\mathrm{f}-\mathrm{Hb}$}

Table 2 shows a comparison of colonoscopic/pathology findings by $\mathrm{f}-\mathrm{Hb}$ concentration. As well as being strongly associated with cancer $(p<0.001)$, a raised $\mathrm{f}-\mathrm{Hb}$ also correlated with the risk of advanced adenoma $(p<0.001)$, any advanced polyp $(p<0.001)$, non-advanced polyps $(p<$ $0.001)$, inflammatory bowel disease $(p<0.001)$ and other malignancy (anal SCC or rectal lymphoma, $p<0.001$ ). There was also a correlation with diverticulosis $(p<0.001)$ although this was predominantly associated with a mildly raised $\mathrm{f}-\mathrm{Hb}(10-149 \mu \mathrm{g} / \mathrm{g})$. Raised $\mathrm{f}-\mathrm{Hb}$ was associated with having any pathology found at colonoscopy $(p<0.001)$; although of interest, $142(20.3 \%)$ patients with a raised $\mathrm{f}-\mathrm{Hb}$ had a completely normal colonoscopy, including 28 $(15.5 \%)$ with a $\mathrm{f}-\mathrm{Hb} \geq 400 \mu \mathrm{g} / \mathrm{g}$.

\section{Raised $\mathrm{f}-\mathrm{Hb}$ Binary logistic regression}

12 variables were chosen for binary logistic regression: age, sex, SIMD, oral anticoagulants, PPI, rectal bleeding, colorectal cancer, advanced adenoma, any advanced polyp, any non-advanced polyp, inflammatory bowel disease and diverticulosis (Table 3). While anal squamous cell carcinoma or rectal lymphoma was found to be significant in $\chi^{2}$ analysis, the absolute number of cases was very small $(n=$ 4) and this could not be included in regression analysis. On univariate analysis older age ( $\geq 75$ years: OR, $1.82(95 \% \mathrm{CI}$ : 1.25-2.64; $p=0.002)$ ), deprivation (SIMD 12: OR, 1.51 (95\% CI: $1.21-1.88 ; p<0.001)$ ), oral anticoagulants (OR, 1.82 (95\% CI: $1.02-3.27 ; p=0.045)$ ), rectal bleeding (OR, 1.50 (95\% CI: $1.20-1.88 ; p<0.001)$ ), colorectal cancer (OR, 8.54 (95\% CI: 3.38-21.57; $p<0.001)$ ), advanced adenoma (OR, 6.68 (95\% CI: 3.57-12.83; $p<0.001)$ ), any advanced polyp (OR, 5.06 (95\% CI: $2.89-8.88 ; p<0.001)$ ), non-advanced polyps (OR, 1.41 (95\% CI: $1.07-1.86 ; p=$ $0.014)$ ) and inflammatory bowel disease (OR, $3.90(95 \%$ CI: $2.07-7.37 ; p<0.001)$ ) correlated with a raised f-Hb. On multivariate analysis, older age ( $\geq 75$ years: OR, $1.52(95 \%$ CI: 1.00-2.32; $p=0.050)$ ), deprivation (SIMD 12: OR, 1.54 (95\% CI: 1.21-1.94; $p<0.001)$ ), oral anticoagulants (OR, 1.78 (95\% CI: $1.01-3.15 ; p=0.046))$, rectal bleeding (OR, 1.47 (95\% CI: $1.15-1.88 ; p=0.002)$ ), colorectal cancer (OR, 9.27 (95\% CI: 3.61-23.83; $p<0.001)$ ), advanced adenoma (OR, 7.52 (95\% CI: 3.90-14.49; $p<0.001)$ ), nonadvanced polyps (OR, 1.78 (95\% CI: 1.33-2.38; $p<0.001)$ ) and inflammatory bowel disease (OR, 4.19 (95\% CI: $2.17-$ 8.07; $p<0.001)$ ) retained significance as independent predictors of a raised $\mathrm{f}-\mathrm{Hb}$.

\section{SBD, other pathology and no pathology}

Next, patients were divided into those with significant bowel disease, other pathology and no pathology. A comparison of these three groups by $\mathrm{f}-\mathrm{Hb}$ concentration can

Table 2. Comparison of colonoscopic/pathology findings by $\mathrm{f}-\mathrm{Hb}$ concentration.

\begin{tabular}{|c|c|c|c|c|c|c|}
\hline & \multirow[b]{2}{*}{ All } & \multicolumn{4}{|c|}{$\mathrm{f}-\mathrm{Hb}$ ( $\mu \mathrm{g} \mathrm{Hb} / \mathrm{g}$ faeces) } & \multirow[b]{2}{*}{$p$} \\
\hline & & $<10$ & $10-149$ & $150-399$ & $\geq 400$ & \\
\hline $\mathrm{N}$ & 1272 & 572 & 430 & 89 & 181 & \\
\hline Significant bowel disease & $223(17.5 \%)$ & $36(6.3 \%)$ & $71(16.5 \%)$ & $34(38.2 \%)$ & $82(45.3 \%)$ & $<0.001$ \\
\hline Colorectal cancer & $54(4.2 \%)$ & $5(0.9 \%)$ & $9(2.1 \%)$ & 7 (7.9\%) & 33 (18.2\%) & $<0.001$ \\
\hline Advanced adenoma & $93(7.3 \%)$ & II (I.9\%) & $42(9.8 \%)$ & $14(15.7 \%)$ & $26(14.4 \%)$ & $<0.001$ \\
\hline Any advanced polyp & 99 (7.8\%) & $15(2.6 \%)$ & $43(10.0 \%)$ & $15(16.9 \%)$ & $26(14.4 \%)$ & $<0.001$ \\
\hline$\geq 5$ polyps & $33(2.6 \%)$ & $6(1.0 \%)$ & 18 (4.2\%) & $5(5.6 \%)$ & $4(2.2 \%)$ & 0.005 \\
\hline IBD & $66(5.2 \%)$ & $12(2.1 \%)$ & $14(3.3 \%)$ & II (I $2.4 \%)$ & $29(16.0 \%)$ & $<0.001$ \\
\hline Other pathology & $682(53.6 \%)$ & $311(54.4 \%)$ & $261(60.7 \%)$ & $39(43.8 \%)$ & 71 (39.2\%) & $<0.001$ \\
\hline Non-advanced polyp & $27 \mathrm{I}(2 \mathrm{I} .3 \%)$ & 104 (18.2\%) & $122(28.4 \%)$ & $16(18.0 \%)$ & $29(16.0 \%)$ & $<0.001$ \\
\hline Other inflammation & $4 I(3.2 \%)$ & $20(3.5 \%)$ & II (2.6\%) & $4(4.5 \%)$ & $6(3.3 \%)$ & 0.750 \\
\hline Diverticulosis & $408(32.1 \%)$ & $172(30.1 \%)$ & $169(39.3 \%)$ & $22(24.7 \%)$ & 45 (24.9\%) & $<0.001$ \\
\hline Haemorrhoids & 197 (I5.5\%) & $100(17.5 \%)$ & $56(13.0 \%)$ & $14(15.7 \%)$ & 27 (14.9\%) & 0.286 \\
\hline Angiodysplasia/Telangiectasia & $10(0.8 \%)$ & $3(0.5 \%)$ & $5(\mathrm{I} .2 \%)$ & $0(0.0 \%)$ & $2(1.1 \%)$ & 0.527 \\
\hline Radiation proctitis & $12(0.9 \%)$ & I (0.2\%) & $8(1.9 \%)$ & I (I.I\%) & $2(1.1 \%)$ & 0.056 \\
\hline Anal SCC or rectal lymphoma & $4(0.3 \%)$ & $0(0.0 \%)$ & $0(0.0 \%)$ & $0(0.0 \%)$ & $4(2.2 \%)$ & $<0.001$ \\
\hline Melanosis coli & II (0.9\%) & $4(0.7 \%)$ & $5(\mathrm{l} .2 \%)$ & I (I.I\%) & I (0.6\%) & 0.825 \\
\hline Anal fissure/Fistula & $8(0.6 \%)$ & $4(0.7 \%)$ & I (0.2\%) & $0(0.0 \%)$ & $3(1.7 \%)$ & 0.191 \\
\hline Rectal prolapse & $3(0.2 \%)$ & $0(0.0 \%)$ & $2(0.5 \%)$ & I (I.I\%) & $0(0.0 \%)$ & 0.126 \\
\hline Fibroepithelial anal polyp & 14 (I.1\%) & $3(0.5 \%)$ & $7(1.6 \%)$ & $3(3.4 \%)$ & I (0.6\%) & 0.056 \\
\hline Lipoma & $4(0.3 \%)$ & $3(0.5 \%)$ & I (0.2\%) & $0(0.0 \%)$ & $0(0.0 \%)$ & 0.626 \\
\hline No pathology & 367 (28.9\%) & 225 (39.3\%) & 98 (22.8\%) & $16(18.0 \%)$ & 28 (15.5\%) & $<0.001$ \\
\hline
\end{tabular}


Table 3. Univariate and multivariate binary logistic regression of factors associated with $\mathrm{f}-\mathrm{Hb} \geq 10 \mu \mathrm{g} / \mathrm{g}$.

\begin{tabular}{|c|c|c|c|c|c|c|c|}
\hline & & \multicolumn{3}{|c|}{ Univariate } & \multicolumn{3}{|c|}{ Multivariate } \\
\hline & & OR & $95 \% \mathrm{Cl}$ & $p$ & OR & $95 \% \mathrm{Cl}$ & $p$ \\
\hline \multirow{3}{*}{ Age (years) } & $<50$ & 1.00 & & & 1.00 & & \\
\hline & $50-74$ & 0.85 & $0.65-1.10$ & 0.220 & 0.82 & $0.61-1.09$ & 0.163 \\
\hline & $\geq 75$ & 1.82 & $1.25-2.64$ & 0.002 & 1.52 & $1.00-2.32$ & 0.050 \\
\hline \multirow[t]{2}{*}{ Sex } & Male & 1.00 & & & & & \\
\hline & Female & 0.81 & $0.65-1.02$ & 0.071 & & & \\
\hline \multirow{2}{*}{ Scottish Index of Multiple Deprivation } & Non-deprived (SIMD 3-5) & 1.00 & & & 1.00 & & \\
\hline & Deprived (SIMD I-2) & 1.51 & $1.21-1.88$ & $<0.001$ & 1.54 & $1.21-1.94$ & $<0.001$ \\
\hline \multirow[t]{2}{*}{ Oral anticoagulants } & No & 1.00 & & & 1.00 & & \\
\hline & Yes & 1.82 & $1.02-3.27$ & 0.045 & 1.78 & $1.01-3.15$ & 0.046 \\
\hline \multirow[t]{2}{*}{ PPI } & No & 1.00 & & & & & \\
\hline & Yes & 1.22 & $0.98-1.52$ & 0.080 & & & \\
\hline \multirow[t]{2}{*}{ Rectal bleeding } & No & 1.00 & & & 1.00 & & \\
\hline & Yes & 1.50 & $1.20-1.88$ & $<0.001$ & 1.47 & $1.15-1.88$ & 0.002 \\
\hline \multirow[t]{2}{*}{ Colorectal cancer } & No & 1.00 & & & 1.00 & & \\
\hline & Yes & 8.54 & $3.38-21.57$ & $<0.001$ & 9.27 & $3.6 I-23.83$ & $<0.001$ \\
\hline \multirow{2}{*}{ Advanced adenoma } & No & 1.00 & & & 1.00 & & \\
\hline & Yes & 6.77 & $3.57-12.83$ & $<0.001$ & 7.52 & $3.90-14.49$ & $<0.001$ \\
\hline \multirow[t]{2}{*}{ Any advanced polyp } & No & 1.00 & & & - & & \\
\hline & Yes & 5.06 & $2.89-8.88$ & $<0.001$ & - & - & 0.484 \\
\hline \multirow[t]{2}{*}{ Any non-advanced polyp } & No & 1.00 & & & 1.00 & & \\
\hline & Yes & $\mathrm{I} .4 \mathrm{I}$ & $1.07-1.86$ & 0.014 & 1.78 & $1.33-2.38$ & $<0.001$ \\
\hline \multirow[t]{2}{*}{ Inflammatory bowel disease } & No & 1.00 & & & 1.00 & & \\
\hline & Yes & 3.90 & $2.07-7.37$ & $<0.001$ & 4.19 & $2.17-8.07$ & $<0.001$ \\
\hline \multirow[t]{2}{*}{ Diverticulosis } & No & 1.00 & & & & & \\
\hline & Yes & 1.18 & $0.93-1.50$ & 0.166 & & & \\
\hline
\end{tabular}

be seen in Table 2. 223 patients were found to have cancer, advanced adenoma, advanced polyps, $\geq 5$ polyps or inflammatory bowel disease (significant bowel disease). 36 $(16.1 \%)$ had f-Hb $<10 \mu \mathrm{g} / \mathrm{g} ; 71(31.8 \%), 10-149 \mu \mathrm{g} / \mathrm{g} ; 34$ (15.2\%), 150-399 $\mu \mathrm{g} / \mathrm{g}$; and $82(36.8 \%), \geq 400 \mu \mathrm{g} / \mathrm{g} .682$ patients were found to have other bowel disease. 311 (45.6\%) had f-Hb $<10 \mu \mathrm{g} / \mathrm{g} ; 261(38.3 \%), 10-149 \mu \mathrm{g} / \mathrm{g} ; 39$ $(5.7 \%), 150-399 \mu \mathrm{g} / \mathrm{g}$; and $71(10.4 \%), \geq 400 \mu \mathrm{g} / \mathrm{g} .367 \mathrm{had}$ no pathology found at colonoscopy. $225(61.3 \%)$ had $\mathrm{f}-\mathrm{Hb}<$ $10 \mu \mathrm{g} / \mathrm{g}$; 98 (26.7\%), 10-149 $\mu \mathrm{g} / \mathrm{g} ; 16$ (4.4\%), 150-399 $\mu \mathrm{g} /$ $\mathrm{g}$; and $28(7.6 \%), \geq 400 \mu \mathrm{g} / \mathrm{g}$. There was a highly significant association between $\mathrm{f}-\mathrm{Hb}$ concentration and increasing 'severity' of colonoscopic findings from no pathology to other pathology to significant bowel disease $(p<0.001)$.

\section{Demographics Associated with Raised $\mathrm{f}-\mathrm{Hb}$ in those with SBD, Other Pathology and No Pathology Binary Logistic Regression}

6 demographics were chosen for binary logistic regression: age, sex, SIMD, oral anticoagulants, PPI and rectal bleeding (Table 4). For those patients with significant bowel disease, only rectal bleeding (OR, 3.63 (95\% CI: 1.66-7.97; $p=$ 0.001 ) correlated with a raised $\mathrm{f}-\mathrm{Hb}$ on univariate analysis. Multivariate analysis was therefore not performed. For those patients with other bowel disease, only PPI use (OR,
$1.60(95 \%$ CI: $1.15-2.11 ; p=0.004))$ correlated with a raised $\mathrm{f}-\mathrm{Hb}$ on univariate analysis. Again, multivariate analysis could not be performed. For those with no pathology, bowel screening age (50-74 years) (OR, 0.55 (95\% CI: $0.36-0.86 ; p=0.009)$ ) predicted lower risk of a raised $\mathrm{f}-\mathrm{Hb}$ and deprivation (SIMD 1-2: OR, 2.12 (95\% CI: $1.38-3.25 ; p=0.001)$ ) predicted a higher risk of raised $\mathrm{f}-\mathrm{Hb}$. On multivariate analysis, bowel screening age (OR, 0.56 (95\% CI: $0.36-0.89 ; p=0.013)$ ) and deprivation (SIMD 1-2: OR 2.13 (95\% CI: 1.38-3.29; $p=0.001)$ ) retained significance as independent predictors of lower and higher risk of a raised $\mathrm{f}-\mathrm{Hb}$, respectively.

\section{Discussion}

To date, no studies have explored demographics independently associated with a raised $\mathrm{f}-\mathrm{Hb}$ in symptomatic patients. In screener participants, a higher $\mathrm{f}-\mathrm{Hb}$ independently correlates with older age, male sex, deprivation, smoking and use of aspirin, NSAIDs, oral anticoagulants, PPIs and antibiotics. $^{22-25}$ In this study, we have shown higher $\mathrm{f}-\mathrm{Hb}$ concentrations are seen in older symptomatic patients $(\geq$ 75 years $)$ but also in younger patients $(<50$ years $)$. This may be related to the impact of bowel cancer screening, with those aged 50-74 years with a raised $\mathrm{f}-\mathrm{Hb}$ being more likely to be investigated via the screener pathway. On multivariate 


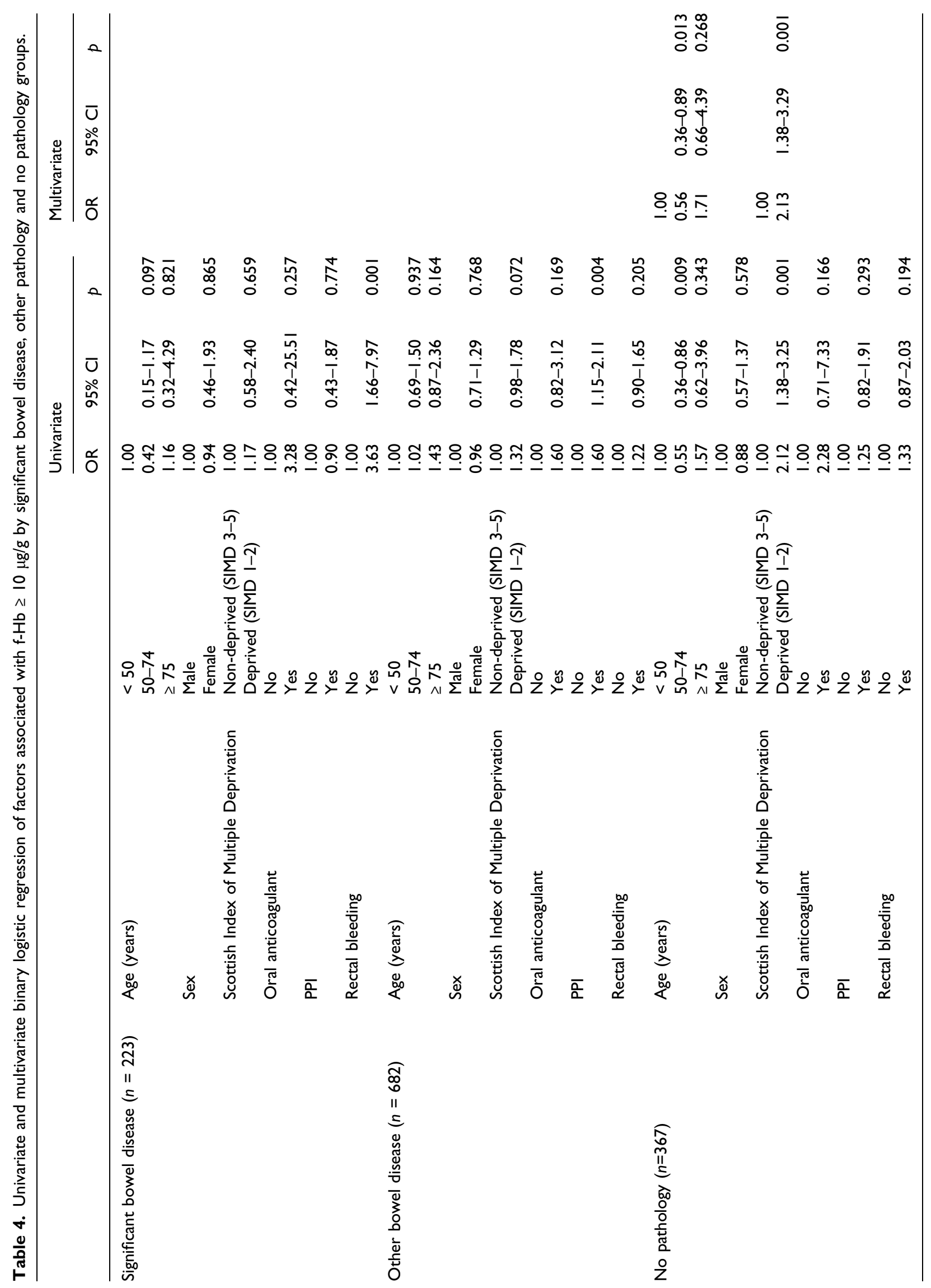


analysis, older age independently predicted a raised $\mathrm{f}-\mathrm{Hb}(p=$ $0.050)$. While in the current study males did constitute a greater proportion of those with a raised $\mathrm{f}-\mathrm{Hb}$ (males accounted for $43.9 \%$ of all participants, $48.1 \%$ of those with $\mathrm{f}-\mathrm{Hb} \geq 400 \mu \mathrm{g} / \mathrm{g}$ and $53.9 \%$ of those with $\mathrm{f}-\mathrm{Hb} 150-399 \mu \mathrm{g} / \mathrm{g}$ ), this did not reach statistical significance $(p=0.080)$. In agreement with studies investigating screener participants, this study has shown deprivation $(p=0.004)$ and oral anticoagulants $(p=$ 0.017 ) to be associated with higher $\mathrm{f}-\mathrm{Hb}$, and these retained significance on multivariate analysis ( $p<0.001$ and $p=0.046$ ). Patients on PPIs were more likely to have a raised $\mathrm{f}-\mathrm{Hb}$, but only between 10 and $399 \mu \mathrm{g} / \mathrm{g}(p=0.007)$. No associations between NSAIDs or aspirin and $\mathrm{f}-\mathrm{Hb}$ were detected.

Several studies have investigated the use of FIT for the diagnosis of significant bowel disease (cancer, advanced adenoma or IBD) in symptomatic patients. McDonald et al. ${ }^{14}$ reported on 280 patients referred from primary care with lower GI symptoms. They found that those with significant bowel disease had a median $\mathrm{f}-\mathrm{Hb}$ of $15 \mu \mathrm{g} / \mathrm{g}$ which was significantly higher than those without $(p<$ $0.0001)$. Additionally, patients with low-risk adenoma had a raised median $\mathrm{f}-\mathrm{Hb}$ of $13 \mu \mathrm{g} / \mathrm{g}$. In a similar study by Godber et al., ${ }^{15}$ of 484 symptomatic patients, 45 had significant bowel disease; 196, low-risk adenoma, hyperplastic polyps, diverticular disease or haemorrhoids; and 243 patients had normal examinations. Median $\mathrm{f}-\mathrm{Hb}$ for each group was $113 \mu \mathrm{g} / \mathrm{g}, 3 \mu \mathrm{g} / \mathrm{g}$ and $2 \mu \mathrm{g} / \mathrm{g}$, respectively $(p<0.0001)$

We have confirmed that in addition to colorectal cancer $(p<0.001)$, advanced adenoma $(p<0.001)$, non-advanced polyps $(p<0.001)$ and inflammatory bowel disease $(p<$ $0.001)$ are all diagnoses independently associated with a raised $\mathrm{f}-\mathrm{Hb}$. We also found diverticulosis to correlate with a mildly raised $\mathrm{f}-\mathrm{Hb}(10-149 \mu \mathrm{g} / \mathrm{g}, p<0.001)$ and a notable association between a raised $\mathrm{f}-\mathrm{Hb}$ and other lower GI malignancies (anal SCC or rectal lymphoma) (all 4 cases $\mathrm{f}-\mathrm{Hb} \geq$ 400, $p<0.001)$. Interestingly, while any advanced polyp (advanced adenoma or advanced sessile serrated polyp) predicted increased $\mathrm{f}-\mathrm{Hb}$ on $\chi^{2}$ analysis $(p<0.001)$ and univariate binary logistic regression $(p<0.001)$, this did not retain significance on multivariate analysis. This most likely reflects the low number of advanced sessile serrated polyps in this study $(n=6)$ but may also relate to previous evidence suggesting that FIT is less sensitive for the detection of sessile serrated polyps as compared to adenoma, which may in part be explained by their frequent proximal colonic location. ${ }^{36,37}$

Several studies have previously examined factors correlating with FIT false positivity in screening participants. In the study by Ibanez-Sanz et al., ${ }^{25} 89,199$ bowel screening FITs from 46,783 patients were reviewed. False positivity was defined as $\mathrm{f}-\mathrm{Hb} \geq 20 \mu \mathrm{g} / \mathrm{g}$ without intermediate-risk, high-risk polyps or cancer. Independent predictors of false positivity were younger age (OR, 1.28 (95\% CI: 1.12-1.46; $p=0.0002)$ ), female sex (OR, 2.31 (95\% CI: 2.03-2.64; $p<$ $0.0001)$ ), successive screening round (OR, 1.53 (95\% CI: $1.35-1.74 ; p<0.0001)$ ), aspirin (OR, 1.30 (95\% CI: 1.04
1.64; $p=0.02)$ ), NSAID (OR, 1.48 (95\% CI: 1.23-1.78; $p<$ $0.0001)$ ), PPI (OR 1.39 (95\% CI: $1.18-1.65 ; p=0.0001)$ ), antibiotics (OR, 1.32 (95\% CI: $1.03-1.71 ; p=0.03))$ and laxative (OR, 2.26 (95\% CI: 1.06-4.80; $p=0.03)$ ) use. Further studies have related false positivity in screening participants to both older age $^{29}$ and younger age, ${ }^{25,30}$ female $25,26,30,32$ and male sex, ${ }^{29}$ smoking, ${ }^{29}$ high BMI, ${ }^{29}$ successive screening, ${ }^{25,26}$ the use of aspirin, ${ }^{25}$ NSAIDs, ${ }^{25}$ PPIs, ${ }^{25,26,31}$ antibiotics $^{25}$ and laxatives, ${ }^{25}$ non-advanced adenomas, ${ }^{27}$ diverticular disease ${ }^{27}$ and anal pathology including haemorrhoids and anal fissures..$^{26,27,29}$ De Klerk et al. ${ }^{28}$ performed a systematic review and meta-analysis of such studies and found younger age, female sex, NSAIDs, PPIs, anal fissures and peptic ulceration to be predictors of FIT false positivity in screener participants.

In the current study, we have established that deprivation is independently associated with a raised $\mathrm{f}-\mathrm{Hb}$ in the absence of pathology at colonoscopy $(p=0.001)$. Mansouri et al., ${ }^{38}$ a co-author of this study, found deprived individuals less likely to have cancer identified as a result of a positive FIT, within the Scottish Bowel Screening programme. It is interesting that this association with deprivation is shared by screening and symptomatic patients. In the review by Barnett et al., ${ }^{39}$ they hypothesise that an elevated systemic inflammatory response (SIR) may explain the higher $\mathrm{f}-\mathrm{Hb}$ concentrations observed in the absence of colorectal pathology, in screener participants with chronic conditions (ischaemic heart disease, cerebrovascular disease, diabetes and hypertension) and on certain medications (PPIs and anticoagulants). Perhaps a heightened SIR is one confounding variable which may link deprivation, co-morbidity and a raised $\mathrm{f}-\mathrm{Hb}$ in the absence of colorectal pathology.

This study has a number of strengths. It is the first to perform multivariate analysis to establish independent predictors of a raised $\mathrm{f}-\mathrm{Hb}$ in patients with lower GI symptoms. While this question has been applied to screener participants, it cannot be assumed that the same associations will be seen in symptomatic patients and indeed we have established several similarities and differences. Our study reflects real-life practice in the GG\&C. Patients with both high- and low-risk symptoms and with and without rectal bleeding were included, reflecting the most up-to-date evidence $^{1,40-42}$ and clinical use of FIT. Our study does however have limitations. It is retrospective in nature, and with the current sample size, it was difficult to establish clear associations between FIT and rarer diagnoses such as angiodysplasia, radiation proctitis, anal SCC, rectal prolapse and sessile serrated adenomas.

\section{Conclusion}

We have found demographics including older age, deprivation and the use of oral anticoagulants to be independently associated with a raised $\mathrm{f}-\mathrm{Hb}$ in patients with lower GI symptoms. In addition to colorectal cancer, advanced 
adenoma, non-advanced polyps, IBD, diverticulosis and anal SCC/rectal lymphoma are associated with a raised $\mathrm{f}-\mathrm{Hb}$. Deprivation is independently associated with a raised $\mathrm{f}-\mathrm{Hb}$ in the absence of pathology. This should be considered when utilising FIT as part of a symptomatic referral pathway. Further work is required to establish why deprived patients are more likely to exhibit a raised $\mathrm{f}-\mathrm{Hb}$ without pathology.

\section{Declaration of conflicting interests}

The author(s) declared no potential conflicts of interest with respect to the research, authorship, and/or publication of this article.

\section{Funding}

The author(s) received no financial support for the research, authorship, and/or publication of this article.

\section{Ethical approval}

Caldicott guardian approval was given by NHS GG\&C to safeguard the record linkage with ethical approval waived for the purposes of service development.

\section{Guarantor}

MJ.

\section{Contributorship}

MJ - conceived the study, data collection, data analysis, reviewed the literature and wrote the manuscript

GM - data collection

GP - data collection

PB - data collection

GK - data collection

JW - reviewed and edited the manuscript

EC - reviewed and edited the manuscript

DM - reviewed and edited the manuscript

PW - reviewed and edited the manuscript

KS - reviewed and edited the manuscript

SM - conceived the study, data analysis, reviewed and edited the manuscript.

\section{ORCID iD}

Mark S. Johnstone (D) https://orcid.org/0000-0002-5035-1517

\section{References}

1. Farrugia A, Widlak M, Evans C, et al. Faecal immunochemical testing (FIT) in symptomatic patients: what are we missing? Frontline Gastroenterol 2020; 11: 28-33. doi:10. 1136/flgastro-2018-101174.

2. Khan AA, Klimovskij M and Harshen R. Accuracy of faecal immunochemical testing in patients with symptomatic colorectal cancer. BJS Open 2020; 4: 1180-1188. doi:10.1002/ bjs5.50346.
3. Westwood M, Lang S, Armstrong N, et al. Faecal immunochemical tests (FIT) can help to rule out colorectal cancer in patients presenting in primary care with lower abdominal symptoms: a systematic review conducted to inform new NICE DG30 diagnostic guidance. BMC Medicine 2017; 15: 189. doi:10.1186/s12916-017-0944-z.

4. Vieito NP, Zarraquiños S and Cubiella J. High-risk symptoms and quantitative faecal immunochemical test accuracy: Systematic review and meta-analysis. World Journal Gastroenterology 2019; 25: 2383-2401. doi:10.3748/wjg.v25. i19.2383.

5. Nicholson BD, James T, Paddon M, et al. Faecal immunochemical testing for adults with symptoms of colorectal cancer attending English primary care: a retrospective cohort study of 14487 consecutive test requests. Aliment Pharmacol Ther 2020; 52: 1031-1041. doi:10.1111/apt.15969.

6. Ayling RM, Machesney M. Service evaluation of faecal immunochemical testing introduced for use in North East London for patients at low risk of colorectal cancer. $J$ Clin Pathol 2021; 74: 163-166. doi:10.1136/jclinpath-2020206632.

7. Westwood M, Corro Ramos I, Lang S, et al. Faecal immunochemical tests to triage patients with lower abdominal symptoms for suspected colorectal cancer referrals in primary care: a systematic review and cost-effectiveness analysis. Health Tech Assess 2017; 21: 1-234. doi:10.3310/hta21330.

8. Mowat C, Digby J, Strachan JA, et al. Faecal haemoglobin concentration thresholds for reassurance and urgent investigation for colorectal cancer based on a faecal immunochemical test in symptomatic patients in primary care. Ann Clin Biochem. 2021.

9. National Institute for Health and Care Excellence. Suspected cancer: recognition and referral [NG12]. London, UK: National Institute for Health and Care Excellence. https:// www.nice.org.uk/guidance/ng12/chapter/1-Recommendationsorganised-by-site-of-cancer\#lower-gastrointestinal-tract-cancers. (accessed 04 September 2021).

10. National Institute for Health and Care Excellence. Quantitative Faecal Immunochemical Tests to Guide Referral for Colorectal Cancer in Primary Care. [DG30]. London, UK: National Institute for Health and Care Excellence. https:/ www.nice.org.uk/guidance/dg30/chapter/1-Recommendations. (accessed 04 January, 2021).

11. Scottish Referral Guidelines for Suspection Cancer. Lower Gastrointestinal Cancer. http://www.cancerreferral.scot.nhs. uk/lower-gastrointestinal-cancer/. http:/www.cancerreferral. scot.nhs.uk/lower-gastrointestinal-cancer/ (accessed 04 September 2021).

12. Digby J, Strachan JA, McCann R, et al. Measurement of faecal haemoglobin with a faecal immunochemical test can assist in defining which patients attending primary care with rectal bleeding require urgent referral. Ann Clin Biochem Int J Lab Med 2020; 57: 325-327. doi:10.1177/0004563220935622.

13. Juul JS, Hornung N, Andersen B, et al. The value of using the faecal immunochemical test in general practice on patients 
presenting with non-alarm symptoms of colorectal cancer. $\mathrm{Br} \mathrm{J}$ Cancer 2018; 119: 471-479. doi:10.1038/s41416-018-0178-7.

14. McDonald PJ, Digby J, Innes C, et al. Low faecal haemoglobin concentration potentially rules out significant colorectal disease. Colorectal Dis 2013; 15: e151-e159. doi: 10. 1111/codi.12087.

15. Godber IM, Todd LM, Fraser CG, et al. Use of a faecal immunochemical test for haemoglobin can aid in the investigation of patients with lower abdominal symptoms. Clin Chem Lab Med (Cclm) 2016; 54: 595-602. doi:10.1515/ cclm-2015-0617.

16. Nakarai A, Hiraoka S, Takahashi S, et al. Simultaneous measurements of faecal calprotectin and the faecal immunochemical test in quiescent ulcerative colitis patients can stratify risk of relapse. J Crohn's Colitis 2017; 12: 71-76. doi: 10.1093/ecco-jcc/jjx118.

17. Shi HY, Chan FKL, Chan AWH, et al. Accuracy of faecal immunochemical test to predict endoscopic and histological healing in ulcerative colitis: a prospective study based on validated histological scores. J Crohn's Colitis 2017; 11: 1071-1077. doi:10.1093/ecco-jcc/jjx088.

18. Kim DJ, Jeoun YM, Lee D-W, et al. Usefulness of fecal immunochemical test and fecal calprotectin for detection of active ulcerative colitis. Intestinal Res 2018; 16: 563-570. doi:10.5217/ir.2018.00020.

19. Dai C, Jiang M, Sun M-J, et al. Fecal immunochemical test for predicting mucosal healing in ulcerative colitis patients: A systematic review and meta-analysis. J Gastroenterol Hepatol 2018; 33: 990-997. doi:10.1111/jgh.14121.

20. Takashima S, Kato J, Hiraoka S, et al. Evaluation of mucosal healing in ulcerative colitis by fecal calprotectin Vs. Fecal immunochemical test. Am J Gastroenterol 2015; 110: 873-880. doi:10.1038/ajg.2015.66.

21. Inokuchi T, Kato J, Hiraoka S, et al. Fecal immunochemical test versus fecal calprotectin for prediction of mucosal healing in crohn's disease. Inflamm Bowel Dis 2016; 22: 1078-1085. doi:10.1097/mib.0000000000000728.

22. Symonds EL, Osborne JM, Cole SR, et al. Factors affecting faecal immunochemical test positive rates: demographic, pathological, behavioural and environmental variables. J Med Screen 2015; 22: 187-193. doi:10.1177/0969141315584783.

23. Digby J, McDonald PJ, Strachan JA, et al. Deprivation and faecal haemoglobin: implications for bowel cancer screening. $J$ Med Screen 2014; 21: 95-97. doi:10.1177/0969141314535388.

24. Clark GRC, Strachan JA, McPherson A, et al. Faecal haemoglobin distributions by sex, age, deprivation and geographical region: consequences for colorectal cancer screening strategies. Clin Chem Lab Med (Cclm) 2020; 58: 2073-2080. doi:10.1515/cclm-2020-0268.

25. Ibáñez-Sanz G, Milà N, de la Peña-Negro LC, et al. Protonpump inhibitors are associated with a high false-positivity rate in faecal immunochemical testing. $J$ Gastroenterol 2021; 56: 42-53. doi:10.1007/s00535-020-01738-z.

26. Ibáñez-Sanz G, Garcia M, Rodríguez-Moranta F, et al. Prescription drugs associated with false-positive results when using faecal immunochemical tests for colorectal cancer screening. Dig Liver Dis 2016; 48: 1249-1254. doi:10.1016/j. dld.2016.06.011.

27. Kim NH, Park JH, Park DI, et al. Are hemorrhoids associated with false-positive fecal immunochemical test results? Yonsei Medical Journal 2017; 58: 150-157. doi:10.3349/ymj.2017. 58.1.150.

28. de Klerk CM, Vendrig LM, Bossuyt PM, et al. Participantrelated risk factors for false-positive and false-negative fecal immunochemical tests in colorectal cancer screening: systematic review and meta-analysis. Am J Gastroenterol 2018; 113: 1778-1787. doi:10.1038/s41395-018-0212-7.

29. Amitay EL, Cuk K, Niedermaier T, et al. Factors associated with false-positive fecal immunochemical tests in a large German colorectal cancer screening study. Int J Cancer 2019; 144: 2419-2427. doi:10.1002/ijc.31972.

30. Symonds EL, Osborne JM, Cole SR, et al. Factors affecting faecal immunochemical test positive rates: demographic, pathological, behavioural and environmental variables. J Med Screen. 2015; 22: 187-193. doi:10.1177/0969141315584783.

31. Rodriguez-Alonso L, Rodriguez-Moranta F, Arajol C, et al. Proton pump inhibitors reduce the accuracy of faecal immunochemical test for detecting advanced colorectal neoplasia in symptomatic patients. PLOS ONE 2018; 13: e0203359. doi:10.1371/journal.pone.0203359.

32. Domper Arnal MJ, García Mateo S, Hermoso-Durán S, et al. False-positive fecal immunochemical test results in colorectal cancer screening and gastrointestinal drug use. Int $J$ Colorectal Dis 2021; 36: 1861-1869. doi:10.1007/s00384-02103947-1.

33. Rutter MD, East J, Rees CJ, et al. British society of gastroenterology/association of coloproctology of Great Britain and Ireland/Public Health England post-polypectomy and post-colorectal cancer resection surveillance guidelines. Gut 2020; 69: 201-223. doi:10.1136/gutjnl-2019-319858.

34. McSorley ST, Digby J, Clyde D, et al. Yield of colorectal cancer at colonoscopy according to faecal haemoglobin concentration in symptomatic patients referred from primary care. Colorectal Dis 2021; 23: 1615-1621. doi: 10.1111/codi. 15405 .

35. World Health Organisation. Haemoglobin Concentrations for the Diagnosis of Anaemia and Assessment of Severity. Vitamin and Mineral Nutritional Information System. Geneva, Switzerland: WHO. https://www.who.int/vmnis/indicators/ haemoglobin.pdf (accessed 23 December 2021).

36. Carot L, Castells A, Hernández C, et al. Detection of serrated lesions in proximal colon by simulated sigmoidoscopy vs faecal immunochemical testing in a multicentre, pragmatic, randomised controlled trial. United Eur Gastroenterol J 2018; 6: 1527-1537. doi:10.1177/2050640618804722.

37. Chang L-C, Shun C-T, Hsu W-F, et al. Fecal immunochemical test detects sessile serrated adenomas and polyps with a low level of sensitivity. Clin Gastroenterol Hepatol 2017; 15: 872-879. 
38. Mansouri D, McMillan DC, Grant Y, et al. The impact of age, sex and socioeconomic deprivation on outcomes in a colorectal cancer screening programme. PloS One 2013; 8: e66063. doi:10.1371/journal.pone.0066063.

39. Barnett KN, Clark GRC, Steele RJC, et al. Faecal haemoglobin estimated by faecal immunochemical tests-an indicator of systemic inflammation with real clinical potential. Diagnostics. 2021; 11: 2093. doi: 10.3390/diagnostics11112093.

40. D'Souza N, Delisle TG, Chen M, et al. Faecal immunochemical testing in symptomatic patients to prioritize investigation: diagnostic accuracy from NICE FIT Study. $B r J$ Surg 2021; 108: 804-810. doi:10.1093/bjs/znaa132.

41. Hicks G, D'Souza N, Georgiou Delisle T, et al. Using the faecal immunochemical test in patients with rectal bleeding: evidence from the NICE FIT study. Colorectal Dis 2021; 23: 1630-1638. doi: 10.1111/codi.15593.

42. Pin-Vieito N, Puga M, Fernández-de-Castro D, et al. Faecal immunochemical test outside colorectal cancer screening? World Journal Gastroenterology 2021; 27: 6415-6429. doi: 10.3748/wjg.v27.i38.6415. 